\title{
Pathogénie du SIDA : \\ I'hypothèse de la mort cellulaire programmée
}

\author{
Jean-Claude Ameisen, André Capron
}

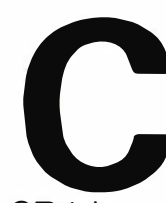

hez le patient infecté par le virus de l'immunodéficience humaine (HIV), la réduction progressive du nombre de lymphocytes $T$ $\mathrm{CD}^{+}+$qui aboutit au SIDA est précédée, pendant plusieurs mois voire des années, par une atteinte fonctionnelle très précoce de cette souspopulation lymphocytaire. Ce dysfonctionnement est caractérisé par une perte sélective de la capacité des lymphocytes T CD4 + à proliférer en réponse à certains stimuli, dont la mobilisation du récepteur $\mathrm{T}(\mathrm{TcR}+)$ par l'anticorps CD3 et par l'ensemble des antigènes mémoires, contrastant avec la persistance d'une capacité au moins partielle de ces cellules à être activées par ces stimuli. Le pourcentage très faible, inférieur à 1 pour 1000 , de lymphocytes T CD4 + infectés par le HIV avant la phase terminale de la maladie a suggéré la nature indirecte de ces anomalies qualitatives précoces et quantitatives tardives, et a conduit à avancer de nombreux mécanismes, parfois contradictoires, pour rendre compte de chacune de ces deux atteintes. Nous avons récemment proposé l'hypothèse [1] qu'un mécanisme unique pourrait être à même, à lui seul, d'expliquer à la fois ces deux anomalies ; il s'agit de la réémergence inappropriée, dans l'ensemble de la souspopulation lymphocytaire $\mathrm{T}$ CD4 + d'un programme de mort cellulaire en réponse à l'activation. La mort cellulaire programmée, ou mort induite par l'activation, ou apoptose, est, contrairement à la nécrose, un processus de mort physiologique qui joue un rôle essentiel dans le renouvellement des tissus embryonnaires et adultes,

\section{TIRÉS A PART}

\section{J.-C. Ameisen}

notamment dans le contrôle de la croissance des cellules souches hématopoiétiques, l'involution thymique et la sélection négative du répertoire $\mathrm{T}$ durant l'ontogénie lymphocytaire, qui participe à l'établissement de la tolérance au soi $([2,3]$ et $m / s$ suppl. au $n^{\circ} 1$, vol. 5 , p. $25 ; n^{\circ} 10$, vol. 5 , p. 788). Ce mécanisme de suicide actif, qui aboutit en quelques heures à la fragmentation de l'ensemble de l'ADN génomique en multiples de 200 paires de bases, représente le mode de réponse normal des thymocytes immatures à la mobilisation de leur TcR [4]. Selon notre hypothèse, la raison pour laquelle les lymphocytes $\mathrm{T}$ $\mathrm{CD} 4$ + de patients HIV + ne prolifèrent pas in vitro en réponse à la mobilisation de leur TcR est que cette stimulation aboutit à leur suicide. In vivo, l'interaction constante des lymphocytes avec les antigènes exprimés par les agents infectieux conduira progressivement, lorsque les capacités de renouvellement du système immunitaire seront dépassées, à une réduction détectable de leur nombre. Il faut noter que toutes les données publiées sur les caractéristiques de la réponse défectueuse des lymphocytes T CD4 + des patients séropositifs à la mobilisation de leur TcR sont compatibles avec les modalités connues de la réponse des thymocytes immatures normaux à ce même stimulus [1]. Seul manque dans le premier cas, à ce jour, toute donnée sur l'existence ou non d'un mécanisme de mort cellulaire en réponse à l'activation. Notre hypothèse peut être expérimentalement testée comme décrit en [1]. Elle implique l'existence de mécanismes indirects par lesquels le HIV pourrait entraîner la réémergence, dans les lymphocytes T CD4 + matures, d'un programme de suicide cellulaire. Des données très récentes recueillies à partir de lymphocytes $\mathrm{T} \mathrm{CD}^{+}{ }^{+}$matures murins
$[5,6]$ montrent qu'il est possible de faire réapparaître dans ces cellules soit par la mobilisation préalable de la molécule CD4, soit en l'absence de cosignaux apportés par les cellules présentatrices d'antigènes - ce type de programme en réponse à une mobilisation du TcR. Ainsi, au cours de l'infection par le HIV, la mobilisation du CD4 par la protéine gp120, par des complexes immuns ou par des auto-anticorps anti-CD4, ou encore les dysfonctions monocytaires qui ont été décrites, pourraient amorcer un programme de mort cellulaire en réponse à une stimulation ultérieure. L'hypothèse prédit qu'à partir du moment où le nombre de cellules infectées dépasse un seuil minimal critique, le taux de délétion lymphocytaire ne sera pas fonction de la quantité de virus produit dans l'organisme, mais du nombre d'antigènes rencontrés. La propriété de certains agents infectieux (staphylocoque, streptocoque, mycoplasme, et peut-être certains rétrovirus tels que le MMTV [7], voire le HIV lui-même), d'exprimer des "superantigènes " capables de stimuler, par une interaction directe avec la chaîne $\mathrm{V} \beta$ du TcR, jusqu'à $30 \%$ des lymphocytes $\mathrm{T} \mathrm{CD} 4{ }^{+}$pourrait aboutir à une amplification importante du processus de délétion. L'hypothèse permet par ailleurs de proposer une explication à deux phénomènes apparemment paradoxaux observés au cours de l'infection par le HIV. Le premier est le très faible nombre de lymphocytes $\mathrm{T}$ CD4 + infectés au cours de la maladie. Étant donné que l'intégration et que l'expression du provirus nécessitent une activation lymphocytaire, si cette activation conduit à un suicide cellulaire rapide, seules les rares cellules qui auront échappé à ce processus seront capables de produire du virus. Le deuxième phénomène est la persistance chez les patients d'une acti- 
vation, voire d'une hyperactivation, des lymphocytes B et T CD8 + cytotoxiques malgré l'absence apparente de fonctions $\mathrm{T} \mathrm{CD}^{+}{ }^{+}$auxiliaires. Or, la mort cellulaire programmée s'accompagne de la libération de certaines lymphokines, dont l'interleukine 2, et de nucléosomes résultant de la fragmentation de l'ADN, capables d'activer le lymphocyte B. Elle pourrait ainsi permettre l'initiation, mais non le contrôle, de l'activation d'autres populations cellulaires.

Enfin, il faut souligner qu'en dehors de l'atteinte du système immunitaire, le SIDA est caractérisé par l'involution progressive, en l'absence de nécrose, de tissus tels que le système nerveux central, les muscles et la moelle osseuse. Nous proposons que, d'une manière générale, la pathogenèse des lentivirus, et en particulier du HIV, est liée à leur capacité de provoquer indirectement, dans certaines populations cellulaires, la dysrégulation des gènes impliqués dans le contrôle des programmes de mort physiologique, et d'accélérer ainsi de manière inappropriée l'involution de ces tissus. Si cette hypothèse était vérifiée, elle pourrait conduire à l'élaboration de stratégies thérapeutiques nouvelles visant à prévenir le SIDA

\section{Jean-Claude Ameisen \\ André Capron \\ CIBP, Inserm U. 167-Cnrs 624, \\ Institut Pasteur, 1, rue Calmette, 59019 Lille, France.}

\section{RÉFÉRENCES}

1. Ameisen JC, Capron A. T-cell dysfiunction and depletion in AIDS : the programmed cell death hypothesis. Immunol Today $1991 ; 4: 102-5$

2. Duvall E, Wyllie AH. Death and the cell. Immunol Today 1986; 7 : 115-9.

3. Goldstein P. Mort cellulaire et système immunitaire. médecine/sciences 1989 ; 5 : 546-53. 4. Blackman M, Kappler J, Marrack P. The role of the T-cell receptor in positive and negative selection of developing $\mathrm{T}$ cells. Science $1990 ; 248$ : 1335-41

5. Newell MK, Haughn LJ, Maroun CR, Julius MH. Death of mature T cells by separate ligation of $\mathrm{CD} 4$ and the $\mathrm{T}$-cell receptor for antigen. Nature 1990 ; 347 : 286-9.

6. Liu Y, Janeway C. Interferon $\gamma$ plays a critical role in induced cell death of effector T cells. J Exp Med 1990 ; 172 : 1735-9.

7. Choi YW, Kappler JW, Marrack P. A superantigen encoded in the open reading frame of the 3' long terminal repeat of mouse mammary tumor virus. Nature 1991； 350 : 203-7. 